\title{
Immunoediting defines prognosis
}

Intertumour and intratumour

genomic heterogeneity are established features of non-smallcell lung cancers (NSCLCs) that, to an extent, account for the variable clinical outcomes of patients. Despite this knowledge, many patients do not respond to treatment as anticipated, and acquired resistance to both novel and more traditional therapeutic interventions remains common. Now, data from the ongoing Tracking Cancer Evolution through Therapy (TRACERx) NSCLC study demonstrate that, similar to genomic heterogeneity, the immunological landscapes of different regions of the same early stage tumour can vary dramatically, which has implications for the prognosis of patients.

First author Rachel Rosenthal explains the main objective of this study: "we wanted to tease apart the impact that the immune microenvironment has on tumour evolution in early stage untreated NSCLC." To do this, a total of 258 samples from different regions of 88 early stage, treatment-naive NSCLCs were examined using histological analyses and RNA sequencing to assess lymphocyte infiltration.

This analysis demonstrated that the majority of tumours have either uniformly low (38 tumours; $43 \%$ ) or uniformly high (25 tumours; 28\%) levels of immune cell infiltration; however, the remaining subset

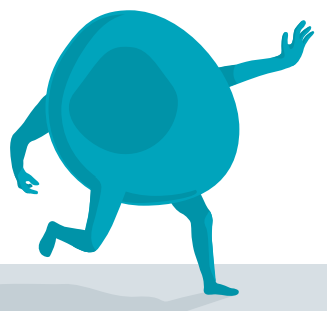

(25 tumours; $28 \%$ ) had disparate levels of immune cell infiltration. Heterogeneous immune cell infiltration was also found to correlate with genomic heterogeneity, including intratumour variations in mutational burden, which might confound several putative biomarkers designed to guide treatment selection.

In order to quantify the extent of 'immunoediting' whereby tumourinfiltrating immune cells might influence tumour evolution, the number of predicted and observed neoantigens was compared. No significant differences between predicted and observed neoantigen levels were detected in DNA from either adenocarcinomas or squamous carcinomas, although reduced germline HLA heterozygosity was associated with fewer neoantigens and immune-low tumours had a significantly increased ratio of observed to expected neoantigens $(P=0.0088)$, implying a reduction in immunoediting. Furthermore, comparisons of spatially distinct samples from the same tumour suggested that up to $42 \%$ of clonal neoantigens might be lost owing to subclonal copy number alterations.

Importantly, RNA sequencing analysis demonstrated that only $33 \%$ of predicted clonal neoantigens were expressed: tumours with high or heterogeneous levels of immune cell infiltration had significantly lower levels of expressed clonal neoantigens than those with limited levels of infiltration (median 29\% and 35\% versus $41 \%$; $P<0.01$ ). Comparisons with non-neoantigenic nonsynonymous mutations confirmed a significant reduction in expressed neoantigens $(P=0.01)$, and this depletion was limited to tumours with intact HLA alleles and, again, to tumours with high or heterogeneous levels of immune cell infiltration, providing further confirmation that these alterations are immune cell mediated. Further investigations of this effect revealed an 11.4-fold increase in promoter methylation of nonexpressed neoantigenic genes relative to their expressed counterparts $(P=0.0016)$, with greater promoter methylation also observed when neoantigenic genes were compared with their non-neoantigenic equivalents (OR 2.3; $P=0.045$ ).

Patients whose tumour material was included in this study had significantly improved disease-free survival outcomes if their tumours had a limited capacity for immune evasion (a phenotype defined by no evidence of immune editing or antigen processing defects and intact HLA alleles) (HR 0.27, 95\% CI 0.12-0.61; $P=0.009$ ), and this prognostic effect was preserved regardless of clonal neoantigen burden.

"We found that the immune landscape can vary greatly within an individual tumour," Rosenthal summarizes, adding "we also identified a number of different mechanisms through which the tumour may evade immune predation, including a novel epigenetic mechanism where transcriptional downregulation of neoantigen encoding genes occurs through promoter hypermethylation."

When asked about future directions, Rosenthal speculates "We want to understand to what extent these mechanisms might play a role in resistance to immunotherapy and whether the dynamic nature of transcriptional downregulation could be exploited in a clinical setting."

Peter Sidaway

ORIGINAL ARTICLE Rosenthal, R. et al. Neoantigen-directed immune escape in lung cancer evolution. Nature 567, 479-485 (2019) 